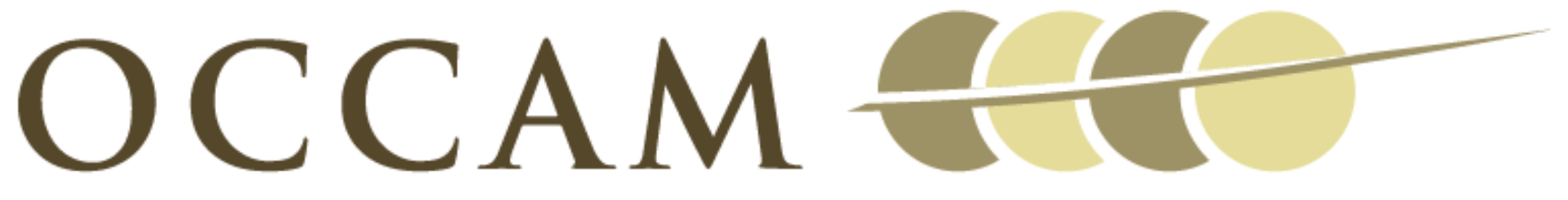

OXFORD CENTRE FOR COLLABORATIVE APPLIED MATHEMATICS

Report Number 11/53

Surface growth kinematics via local curve evolution

by

Derek E. Moulton and Alain Goriely

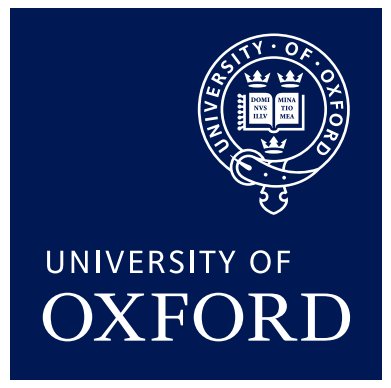

Oxford Centre for Collaborative Applied Mathematics Mathematical Institute

24 - 29 St Giles'

Oxford

OX1 3LB

England 



\title{
Surface growth kinematics via local curve evolution
}

\author{
Derek E. Moulton • Alain Goriely
}

Received: date / Accepted: date

\begin{abstract}
A mathematical framework is developed to model the kinematics of surface growth for objects that can be generated by evolving a curve in space, such as seashells and horns. Growth is dictated by a growth velocity vector field defined at every point on a generating curve. A local orthonormal basis is attached to each point of the generating curve and the velocity field is given in terms of the local coordinate directions, leading to a fully local and elegant mathematical structure. Several examples of increasing complexity are provided, and we demonstrate how biologically relevant structures such as logarithmic shells and horns emerge as analytical solutions of the kinematics equations with a small number of parameters that can be linked to the underlying growth process. Direct access to cell tracks and local orientation enables for connections to be made to the underlying growth process.
\end{abstract}

Keywords Biological growth · morphology $\cdot$ seashell $\cdot$ mathematical model

\section{Introduction}

Surface growth, or accretive growth, refers to the deposition of mass on the surface of a body. Surface growth appears in many physical, biological, and industrial processes, including planetary accretion (Pollack et al 1996), cell motility (Hodge and Papadopoulos 2011), secondary growth in trees (Fournier et al 1994), and industrial surface coating (Tsui and Clyne 1997), as well as

D.E. Moulton

OCCAM, Mathematical Institute

University of Oxford, Oxford, UK

E-mail: moulton@maths.ox.ac.uk

A. Goriely

OCCAM, Mathematical Institute

University of Oxford, Oxford, UK 
several biological growth processes outlined below. From a continuum mechanics standpoint, surface growth poses a particular challenge. Since new material points are continually deposited on the surface of the body, material points in the current configuration of the body cannot all be mapped back to a unique reference configuration, and so incorporating surface growth within classical continuum mechanics requires particular care (Garikipati 2009). Hence, there is significant interest in the topic from a fundamental mechanical standpoint as well, and several theories and continuum balance laws have recently been proposed to address this issue (Ateshian 2007; Hodge and Papadopoulos 2010; Epstein 2010).

In a certain class of surface growth, mass is deposited onto the surface of a body that is not easily deformed. We classify this as hard body accretion, and in such a case elasticity of the body becomes negligible. This is to be distinguished from soft body accretion, in which elastic deformations are non negligible and the relationship between growth and stress is an important factor. Examples of bodies which grow via hard body accretion include seashells, bones, horns, antlers, and teeth. Growth takes place by adding mass locally on the boundary of the structure and there is no possibility of deforming the material in the bulk to add mass. Considering shells, horns, and antlers, some very interesting and diverse structures are created through this process, with multiple levels of patterning and often resulting in self-similar structures. It is remarkable that a local process results in such beautiful, mathematically elegant global structures. A natural question that we address here is how local deposition of mass can result in regular global structures.

The patterns inherent in these surfaces have long been recognised and appreciated, and have inspired a large body of research focused on morphology. This is particularly true in the case of seashells. Initial efforts date back to 1838 (Moseley 1838) and include the classic work of D'Arcy Thompson (Thompson 1942), which described the elegance and simplicity of the spiral coils of the molluscan shell in mathematical and geometric terms. Following the work of Raup (Raup 1961; Raup and Michelson 1965), who triggered the field of research termed "theoretical morphology," many models and approaches have been devised. A description and classification of various models and approaches can be found in (Stone 1996; Dera et al 2008). While most of the early works were descriptive, focusing on the form and shape of shells, research has shifted in recent decades to questions of biology and evolution (Rice 1998), understanding pigmentation patterns (Boettiger et al 2009; Meinhardt 2009), and the growth process underlying shell form (Hammer and Bucher 2005). Nevertheless, even in the case of the molluscan shell, which has been reproduced mathematically numerous times, a full understanding of how growth occurs, e.g. the biomechanical basis for coiling, remains elusive. In this paper we develop a mathematical framework suitable to address these questions.

There are several key elements present in our formulation, and it is instructive to elucidate these in comparison to previous work. One element is the use of growth velocity vectors. In our model, accretive growth occurs by the evolution of a generating curve, characterised by a deposition rate and a spatial 
direction. The direction and rate of deposition are described by a vector field defined at every point on the generating curve. The formulation has general applicability, allowing for arbitrary growth velocities and without assumption on the shape of the generating curve. The analysis naturally lends itself to a discussion of cell tracks, i.e. the path of cells all created by the same material point on the generating curve. This is similar to the model of Skalak et al. in 1997 (Skalak et al 1997). These authors showed how a variety of different biologically relevant structures could be created through the proper choice of growth vector field. However, another key element in our work is a purely local description. The growth velocities in (Skalak et al 1997) were defined in terms of a fixed coordinate system. Here, we define a local orthonormal frame on the generating curve, and the growth vector field is defined in terms of the local frame and local geometry. In this manner, the evolution of the curve as well as the form of the growth vector field are directly connected to local information and the intrinsic geometry of the surface. The idea is that in an actual biological growth process, the mechanisms controlling the accretion and rate of the deposition of material do not depend on the global structure or on global positional coordinates, but rather on the local geometry and local orientation. Thus, to understand and model the underlying mechanisms in the growth process, a local, intrinsic description of growth is needed.

The notion and benefit of a local rather than a global description has been previously noted for seashells. The idea was initially proposed by Okamoto (Okamoto 1988a,b), who attached a Frenet frame to a centerline curve of tubular shells, and simulated previously inaccessible shells by varying the expansion of the tube and the curvature and torsion of the centerline curve. Several other models have since utilised the same approach (Savazzi 1987, 1990; Ackerly 1989; Tyszka and Topa 2005). These models have the advantage that they do not rely on an artificial fixed frame, however this description does not incorporate the actual growth process associated with material deposition rates. More recent models use growth vectors defined on an aperture (Hammer and Bucher 2005; Urdy et al 2010). Such models can create fairly general shell evolutions and explicitly include the aspect of time in shell growth; however, they are still largely based on global definitions and typically cannot provide a convenient mathematical description in terms of a minimal number of parameters.

The purpose of the present work is to formulate a model of accretive growth that is both fully local, generally applicable, and analytically tractable. To do so, we start with a local description by attaching an orthonormal frame to each point of a generating curve (rather than a centerline curve, itself an artificial construction). We show that such an approach leads to a mathematically elegant formulation, as we are able to utilise well developed concepts from differential geometry of curves. Hence, quantities such as curvature and twist arise naturally. Moreover, we are not bound to computer algorithms to generate surfaces; rather we can in certain cases achieve analytical descriptions of complex surfaces in terms of a few parameters directly connected to material deposition rates, leading to a qualitative description of surface growth. 
The goal here is not to study a particular structure (e.g. a spiral shell), but rather to start with simple structures and gradually incorporate more complexity, building up local rules and relationships that can generally be applied and connected to growth mechanisms. Doing so, we identify how local growth rules are related to global geometry in a way that can be connected to underlying mechanics and biology. The model we develop is generally applicable to surface growth processes. Given the large body of research and the numerous mathematical models that have been devoted to seashell form and growth, it is most natural to interpret our results in this context, and hence we apply our analysis in particular to understanding shell growth.

Note that our approach consists of the creation of surfaces through the evolution of a generating curve, as opposed to a generating surface. Hence, our analysis relates to structures whose cross section can be modelled as a two-dimensional curve. That is, these structures have a transverse length scale much smaller than the other two typical length scales. Biologically, the analysis is connected to seashells and horns (which are hollow), as opposed to teeth and antlers (which are not hollow).

This paper is organised as follows: in Section 2 we formulate the framework and the equations modelling the accretive growth process. In Section 3 we give several examples utilising a circular generating curve to illustrate the computational steps and develop relationships between geometry and the local velocity field. In Section 4, we consider an arbitrary generating curve and derive conditions on the local velocity for the shape to remain constant during growth. We also connect the intrinsic growth process to an extrinsic approach for surface generation. Conclusions and interpretations with regards to mollusc shells are given in Section 5.

\section{Description of accretive growth}

To model surface accretion, we consider the growth of a surface from a generating curve. We assume that at time $t=0$, we have a curve $\mathbf{r}_{0}(S)=\mathbf{r}(S, 0)$ : $[0, L] \rightarrow \mathbb{R}^{3}$ defined for a material parameter $S$. Accretion is modeled by the evolution of this initial curve. Together with the initial curve, we define at each point on the curve a vector field $\mathbf{q}(S, 0)$ representing the local direction and rate of growth at each material point $S$ (See Fig.1). In general, we specify a velocity field $\mathbf{q}(S, t)$ defining the growth velocity of material point $S$ at time $t$. The velocity field directs the evolution of the generating curve and defines a surface $\mathbf{r}(S, t)$. The curve along time for a fixed $S$ will be referred to as a cell track, that is the track generated by a fixed an initial material point. We now wish to describe the velocity field as a local process. It is reasonable to assume that the velocity vector is a function of the local geometry as well as possible physical, chemical, or biological fields (rate of accretion, morphogen gradient, temperature, $\mathrm{pH}$, etc...). In order to describe the velocity locally, we attach to the curve $\mathbf{r}(S, t)$ a local frame

$$
\mathbf{D}=\left(\begin{array}{lll}
\mathbf{d}_{1} & \mathbf{d}_{2} & \mathbf{d}_{3}
\end{array}\right)
$$




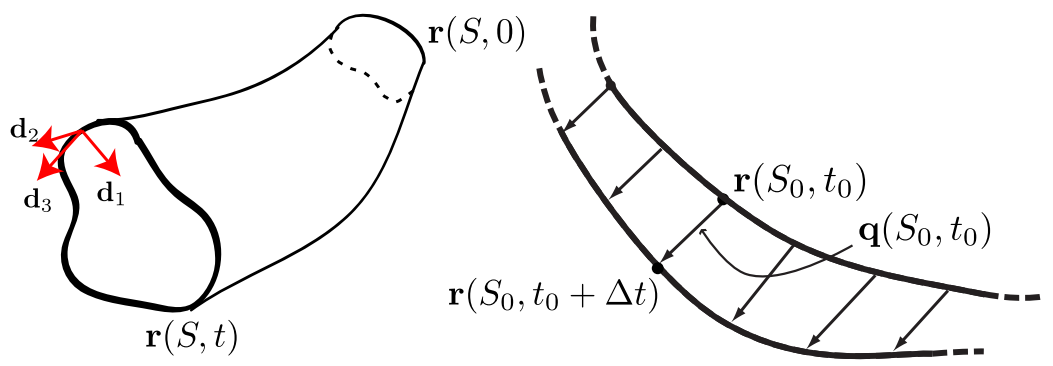

Fig. 1 Creation of a surface from an initial generating curve.

composed of 3 orthonormal vectors $\mathbf{d}_{i}, i=1,2,3$ (see Figure 1 ), with the property that the unit vector $\mathbf{d}_{3}$ is the unit tangent vector related to the spatial derivative of $\mathbf{r}(S, t)$ by a stretch $\lambda$,

$$
\mathbf{r}^{\prime} \equiv \partial_{S} \mathbf{r}(S, t)=\mathbf{D} \cdot \boldsymbol{\Lambda}=\lambda \mathbf{d}_{3}
$$

where $\boldsymbol{\Lambda}=(0,0, \lambda)$ describes the stretch of the curve with respect to the initial arc length, expressed in the local frame. The general basis $\mathbf{D}$ forms an orthonormal basis for all $S$ and $t$, and thus derivatives of the frame can be expressed in terms of the frame, that is

$$
\begin{aligned}
& \mathbf{D}^{\prime} \equiv \partial_{S} \mathbf{D}=\mathbf{D} \cdot \mathbf{U}, \\
& \dot{\mathbf{D}} \equiv \partial_{t} \mathbf{D}=\mathbf{D} \cdot \mathbf{W}
\end{aligned}
$$

Here $\mathbf{U}$ is the Darboux matrix, a skew-symmetric matrix describing the rotation of the local basis at a given time $t$ along the curve with axial vector $\mathrm{u}=\left(\mathrm{u}_{1}, \mathrm{u}_{2}, \mathrm{u}_{3}\right)^{1}$, and $\mathbf{W}$ is the spin matrix, a skew-symmetric matrix describing the rotation of the local basis at a point $S$ as time evolves with axial vector $\mathrm{w}=\left(\mathrm{w}_{1}, \mathrm{w}_{2}, \mathrm{w}_{3}\right)$. Each component $\mathrm{u}_{i}$ describes the rotation of the frame around the basis vector $\mathbf{d}_{i}$ in space, while the $w_{i}$ 's describe the rotation of the frame around the $\mathbf{d}_{i}$ in time. Explicitly, the matrices $\mathbf{U}$ and $\mathbf{W}$ read

$$
\mathbf{U}=\left(\begin{array}{ccc}
0 & -\mathrm{u}_{3} & \mathrm{u}_{2} \\
\mathrm{u}_{3} & 0 & -\mathrm{u}_{1} \\
-\mathrm{u}_{2} & \mathrm{u}_{1} & 0
\end{array}\right), \quad \mathbf{W}=\left(\begin{array}{ccc}
0 & -\mathrm{w}_{3} & \mathrm{w}_{2} \\
\mathrm{w}_{3} & 0 & -\mathrm{w}_{1} \\
-\mathrm{w}_{2} & \mathrm{w}_{1} & 0
\end{array}\right)
$$

Given a curve, its attached general basis is determined up to an arbitrary choice of angle $\varphi$ between the normal vector to the curve and the vector $\mathbf{d}_{1}$. If $\varphi=0$, we have the Frenet-Serret frame defined by the triple $\left(\mathbf{d}_{1}, \mathbf{d}_{2}, \mathbf{d}_{3}\right)=(\boldsymbol{\nu}, \boldsymbol{\beta}, \boldsymbol{\tau})=($ normal, binormal, tangent). In general, the vectors $\left(\mathbf{d}_{\mathbf{1}}, \mathbf{d}_{\mathbf{2}}\right)$ lie in the normal plane to the tangent and are related to the normal and binormal vectors by

$$
\begin{aligned}
& \mathbf{d}_{1}=\boldsymbol{\nu} \cos \varphi+\boldsymbol{\beta} \sin \varphi \\
& \mathbf{d}_{2}=-\boldsymbol{\nu} \sin \varphi+\boldsymbol{\beta} \cos \varphi .
\end{aligned}
$$

1 Following (Antman 1995), we use the sans-serif fonts to denote the components of a vector in the local basis, that is $\mathbf{u}=\mathbf{u}_{1} \mathbf{d}_{1}+\mathbf{u}_{2} \mathbf{d}_{2}+\mathbf{u}_{3} \mathbf{d}_{3}$. 
Note also that the elements of $\mathbf{U}$ are directly related to the usual notion of curvature and torsion. Once the generating curve and its attached basis are known, we express the velocity vector $\mathbf{q}(S, t)$ in terms of the local frame,

$$
\dot{\mathbf{r}}(S, t) \equiv \partial_{t} \mathbf{r}=\mathbf{q}(S, t)=\mathbf{D} \cdot \mathbf{q} .
$$

Here, $\mathbf{q}=\left(\mathrm{q}_{1}, \mathrm{q}_{2}, \mathrm{q}_{3}\right)$ is the velocity vector expressed in the local frame as opposed to $\mathbf{q}$, which is the velocity in a reference frame. Since the objective is to describe the velocity locally, we restrict our attention to q. Assuming that the curve $\mathbf{r}$ is at least twice differentiable in $t$ and $S$, we have two sets of compatibility conditions

$$
\begin{aligned}
& \partial_{S}\left(\partial_{t} \mathbf{r}\right)=\partial_{t}\left(\partial_{S} \mathbf{r}\right), \\
& \partial_{S}\left(\partial_{t} \mathbf{D}\right)=\partial_{t}\left(\partial_{S} \mathbf{D}\right) .
\end{aligned}
$$

Inserting (2) and (8) into (9), and (3) and (4) into (10), and using the fact that the general basis $\mathbf{D}$ is orthonormal (that is, $\mathbf{D}^{\mathrm{T}} \cdot \mathbf{D}=\mathbf{1}$ ), we obtain, after simplification

$$
\begin{aligned}
& \mathbf{q}^{\prime}-\dot{\Lambda}=\mathbf{W} \cdot \boldsymbol{\Lambda}-\mathbf{U} \cdot \mathbf{q}, \\
& \mathbf{W}^{\prime}-\dot{\mathbf{U}}=\mathbf{U} \cdot \mathbf{W}-\mathbf{W} \cdot \mathbf{U} .
\end{aligned}
$$

In components, these two equations read

$$
\begin{aligned}
& \mathrm{q}_{1}^{\prime}+\mathrm{u}_{2} \mathrm{q}_{3}-\mathrm{u}_{3} \mathrm{q}_{2}=\lambda \mathrm{w}_{2} \\
& \mathrm{q}_{2}^{\prime}+\mathrm{u}_{3} \mathrm{q}_{1}-\mathrm{u}_{1} \mathrm{q}_{3}=-\lambda \mathrm{w}_{1} \\
& \mathrm{q}_{3}^{\prime}+\mathrm{u}_{1} \mathrm{q}_{2}-\mathrm{u}_{2} \mathrm{q}_{1}=\dot{\lambda} \\
& \dot{\mathrm{u}}_{1}-\mathrm{w}_{1}^{\prime}=\mathrm{u}_{2} \mathrm{w}_{3}-\mathrm{u}_{3} \mathrm{w}_{2} \\
& \dot{\mathrm{u}}_{2}-\mathrm{w}_{2}^{\prime}=\mathrm{u}_{3} \mathrm{w}_{1}-\mathrm{u}_{1} \mathrm{w}_{3} \\
& \dot{\mathrm{u}}_{3}-\mathrm{w}_{3}^{\prime}=\mathrm{u}_{1} \mathrm{w}_{2}-\mathrm{u}_{2} \mathrm{w}_{1} .
\end{aligned}
$$

For a given velocity vector $\mathbf{q}$, these equations form a set of 6 nonlinear first order partial differential equations for the 7 dependent variables $\mathbf{u}, \mathbf{w}, \lambda$. The extra degree of freedom is given by the choice of the general basis. If for instance, one chooses, the Frenet frame, then $\mathbf{d}_{1}$ is along the normal and $\mathbf{u}_{1}=0$ which reduces the equations to a set of 6 equations for 6 unknowns which can in theory be solved for given initial data. Once $\mathbf{u}(S, t)$ and $\lambda$ are known, the surface built through the accretive process is obtained by integrating the system of 12 nonlinear ODEs given by the Frenet equations $\partial_{S} \mathbf{D}=\mathbf{D} \cdot \mathbf{U}$ together with $\partial_{S} \mathbf{r}(S, t)=\lambda \mathbf{d}_{3}$. Alternatively, and as we shall see, more practically, the surface may be obtained from $\mathbf{w}(S, t)$ and $\lambda$ by integrating $\partial_{t} \mathbf{D}=\mathbf{D} \cdot \mathbf{W}$ together with $\partial_{t} \mathbf{r}(S, t)=\mathbf{D} \cdot \mathbf{q}$. 
2.1 On the choice of frame

Within this intrinsic growth description, there are two ways to view the local velocity field q. Assuming that the surface is known, that is given a parameterised surface and a choice of basis, one could work backwards and determine q. We explore this idea in Section 4.2.

The forward direction is to choose a frame, provide a structure for the local velocity field, and solve the system to determine the full surface. The challenge is in defining appropriate local velocity rules. In doing this, the benefit is that connections can be made to the biological processes underlying growth. Since the local velocity field is defined on the local frame, the choice of frame can be very important. We contend that, unlike the case of elastic rods (Antman 1995), for many surface growth processes, the Frenet frame is the most reasonable. The reason is that in many accretive growth processes, the generating curve is planar or very nearly planar, and for a planar curve to evolve and generate a 3D surface, there must be a component of growth in the direction normal to the plane of the curve; i.e. the binormal direction. The Frenet frame is ideally suited to describe growth in the binormal direction. Note that for the Frenet frame, the Darboux matrix $\mathbf{U}$ takes the form

$$
\mathbf{U}=\lambda\left(\begin{array}{ccc}
0 & -\tau & \kappa \\
\tau & 0 & 0 \\
-\kappa & 0 & 0
\end{array}\right)
$$

where $\kappa \geq 0$ and $\tau$ are the usual geometric curvature and torsion.

\section{Examples}

To understand the computational steps involved in solving this system of equations, we consider a few examples.

\subsection{Straight cylinder}

We first consider the case of growing a straight cylinder. We take the generating curve $\mathcal{C}_{0}$ to be a circle of radius $1, \mathbf{r}(S, 0)=[\cos (S), \sin (S), 0]$, and we choose the general basis to be the Frenet basis and a constant velocity field along the binormal, that is $\mathbf{q}=(0,1,0)$. In this simple case, the compatibility equations and frame evolution are trivial, and the surface is found by integrating (8), i.e.

$$
\mathbf{r}(S, t)=\int_{0}^{t} \mathbf{q} d t+\mathbf{r}(S, 0)=[\cos (S), \sin (S), t]
$$

producing, as expected, a parameterisation of a cylinder. 
3.2 Twisted cylinder, cone

Next, we add a constant velocity component in the tangential and normal directions. We take $\mathcal{C}_{0}$ to be the same circle of radius 1 , and consider the velocity field $\mathbf{q}=\left(c_{1}, c_{2}, c_{3}\right)$, where the $c_{i}$ are constants. In this case, the generating curve remains a circle for all time, and the components of the Darboux vector are $\mathbf{u}_{1}=\mathrm{u}_{3}=0, \mathrm{u}_{2}=\lambda / a$, where $a$ is the circle radius.

Suppose $c_{1}=0$, with $c_{2}, c_{3} \neq 0$. Equation (15) implies $\dot{\lambda}=0$, thus $\lambda \equiv 1$. That is, with no velocity in the normal direction, the circle does not stretch. It follows that $\mathrm{u}_{2} \equiv 1$. Next, (14) implies $\mathrm{w}_{1}=0$, which then implies $\mathrm{w}_{3}=0$ via (16). Then, from (13) we have $w_{2}=c_{3}$ (Equations (17) and (18) are automatically satisfied). Thus, the axial vector is $\mathbf{w}=\left(\mathrm{w}_{1}, \mathrm{w}_{2}, \mathrm{w}_{3}\right)=\left(0, c_{3}, 0\right)$ and the frame evolution equation $\partial_{t} \mathbf{D}=\mathbf{D} \cdot \mathbf{W}$ reads

$$
\begin{aligned}
\dot{\mathbf{d}}_{1} & =-c_{2} \mathbf{d}_{3}, \\
\dot{\mathbf{d}}_{2} & =0, \\
\dot{\mathbf{d}}_{3} & =c_{2} \mathbf{d}_{1},
\end{aligned}
$$

along with initial conditions $\mathbf{d}_{1}(S, 0)=[-\cos (S),-\sin (S), 0]^{T}, \mathbf{d}_{2}(S, 0)=$ $[0,0,1]^{T}, \mathbf{d}_{3}(S, 0)=[-\sin (S), \cos (S), 0]^{T}$. The surface is then obtained by integrating $\partial_{t} \mathbf{r}(S, t)=\mathbf{D} \cdot \mathbf{q}$ with initial conditions $\mathbf{r}(S, 0)=[\cos (S), \sin (S), 0]^{T}$. This yields

$$
\mathbf{r}(S, t)=\left[\begin{array}{c}
\cos \left(S+c_{3} t\right) \\
\sin \left(S+c_{3} t\right) \\
c_{2} t
\end{array}\right]
$$

This is also a parameterisation of a cylinder, but different than (20), as can be understood in terms of the cell tracks. In (22) there is a tangential component to the velocity, leading to twisted cell tracks, whereas in (20) cell tracks are all vertical lines.

Now we add a normal velocity component, so that each $c_{i} \neq 0$. Even though the size of the circle will vary over time due to the normal velocity, $\mathrm{u}_{2}$ is constant. To see this, note that if at time $t$ the radius is $a$, then $\lambda=a$ and $\kappa=1 / a$, thus $\mathrm{u}_{2}=\lambda \kappa=1$. The compatibility equations are solved similarly, except that now $\dot{\lambda}=-c_{1}$, implying $\lambda=\lambda_{0}-c_{1}$. Then, from Equation (13) we have $\mathrm{w}_{2}=c_{3} / \lambda$, and as before $\mathrm{w}_{1}=\mathrm{w}_{3}=0$. Recall that $\mathrm{w}_{2}$ describes the rate that the local frame rotates about the binormal direction. That $w_{2}$ is a function of time relates the fact that for the cells to move tangentially around the circle with constant velocity while the circle changes size, the rotation rate of the frame must slow down as the radius increases. Alternatively, a constant rotation rate of the frame, i.e. $\mathrm{w}_{2}$ constant, can be accomplished by taking $\mathrm{q}_{3}=c_{3} \lambda$. In this case, the frame evolution is the same as the twisted cylinder 


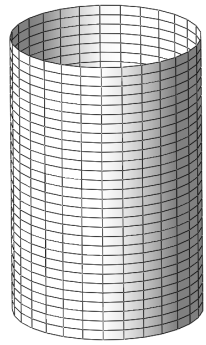

$\mathrm{q}_{1}=0, \mathrm{q}_{2}=1, \mathrm{q}_{3}=0$

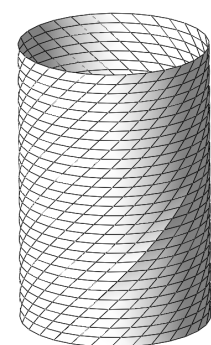

$\mathrm{q}_{1}=0, \mathrm{q}_{2}=1, \mathrm{q}_{3}=1$

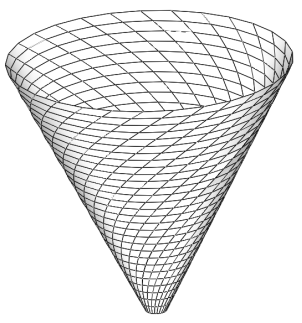

$\mathrm{q}_{1}=-0.5, \mathrm{q}_{2}=1, \mathrm{q}_{3}=0.1 \lambda$

Fig. 2 A straight cylinder (left), twisted cylinder (middle), and twisted cone (right), and the local velocity fields which produced them, expressed in the Frenet frame.

case, and the surface parameterisation is

$$
\mathbf{r}(S, t)=\left[\begin{array}{c}
\lambda \cos \left(S+c_{3} t\right) \\
\lambda \sin \left(S+c_{3} t\right) \\
c_{2} t
\end{array}\right]
$$

with $\lambda=1-c_{1} t$. The form is similar to the twisted cylinder (22), but with the added scale factor for the linearly increasing radius, and the resulting surface is a cone with twisted cell tracks. Figure 2 shows the straight cylinder, twisted cylinder, and twisted cone cases for particular values of the constants. Observe that for the twisted cone, the tangential velocity is smaller near the bottom of the cone, in accordance with the constant rotation rate.

\subsection{Torus}

The previous examples were particularly simple in that the velocity field was constant in space and thus the center point of the generating circle followed a straight line. As a slightly more complex example, we grow a torus. We again take take $\mathbf{r}(S, 0)=[\cos (S), \sin (S), 0]$. Growing a torus requires nonuniform growth in the binormal direction to cause a coiling of the curve. For the Frenet basis, coiling is achieved by taking $\mathrm{q}_{2}$ to be a linear function along an axis in the plane of the generating curve. We will refer to this axis as the growth axis. Then the curve will rotate about a line perpendicular to the growth axis within the plane of the generating curve. Choosing, for instance, the $x$-axis of the initial curve, the velocity is expressed by

$$
\mathrm{q}_{2}=b_{1}+b_{2} \cos (S),
$$

where $b_{1}$ and $b_{2}$ are constants, and the curve will rotate around the line $x=$ $-b_{1} / b_{2}$. How quickly the surface rotates is captured by the constant $b_{2}$. The 

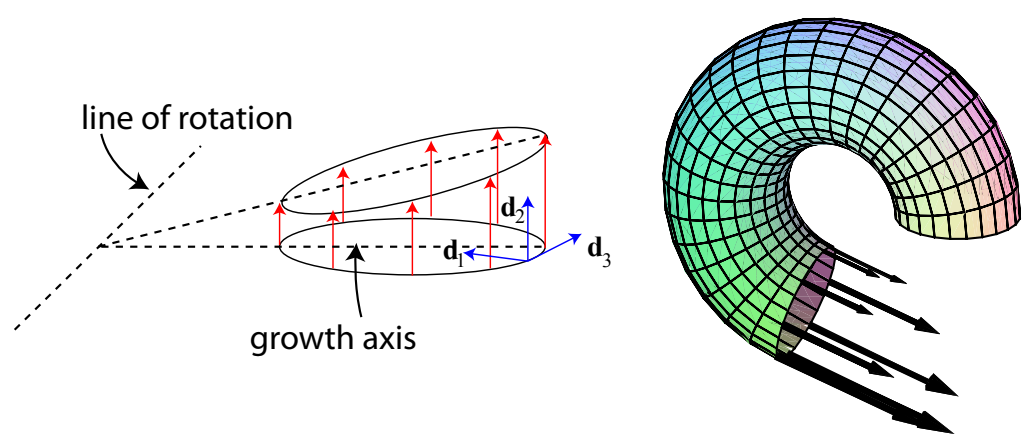

Fig. 3 Growth of a torus. On the left is a schematic illustrating the linear growth along the growth axis. On the right is a growing torus along with velocity field on the generating curve.

form (24) follows from the requirement that the generating curve remains a circle. (This is a consequence of a general result derived in Section 4.1.)

Since the generating curve remains a circle, it follows that $u_{1}=u_{3}=0$ and $\mathrm{u}_{2}=1$. For $\mathrm{q}_{2}$ given by Equation (24) and with $\mathrm{q}_{1}=\mathrm{q}_{3}=0$, we find from the compatibility equations that

$$
\mathrm{w}_{1}=b_{2} \sin (S), \mathrm{w}_{2}=0, \mathrm{w}_{1}=-b_{2} \cos (S) .
$$

The accretive surface may now be found by the same steps as in the previous examples, by first determining the evolution of the frame $\mathbf{D}$ through solving the system $\partial_{t} \mathbf{D}=\mathbf{D} \cdot \mathbf{W}$ and then integrating $\partial_{t} \mathbf{r}(S, t)=\mathbf{D} \cdot \mathbf{q}=\mathbf{q}_{2} \mathbf{d}_{2}$ with initial condition $\mathbf{r}(S, 0)=[\cos (S), \sin (S), 0]^{T}$. We find, after some simplifications:

$$
\mathbf{r}(S, t)=\left[\begin{array}{c}
\frac{1}{b_{2}}\left(\cos \left(b_{2} t\right) \mathbf{q}_{2}-b_{1}\right) \\
\sin (S) \\
\frac{\mathrm{q}_{2}}{b_{2}} \sin \left(b_{2} t\right)
\end{array}\right]
$$

where $\mathrm{q}_{2}=b_{1}+b_{2} \cos (S)$. In Figure 3 we plot the growing torus for $b_{1}=$ $2, b_{2}=1$; included is a schematic to illustrate how the growth gradient causes rotation.

\subsection{Curvilinear horns/shells}

Given the local velocity field structure for rotating a curve as developed in the previous subsection, we can easily extend this construction to more complex and biologically relevant accretive surfaces. Suppose that we add to the torus formulation constant growth in the normal direction, i.e. $q_{1}=-c$. There are three basic ways to combine this with the binormal growth $\mathrm{q}_{2}$ in Equation (24). As described, the form $\mathrm{q}_{2}=b_{1}+b_{2} \cos (S)$ with constant $b_{i}$ defines a 
linear growth profile along a growth axis (the $x$-axis of the initial curve as we have defined it). For the velocity field

$$
\mathrm{q}_{1}=-c, \mathrm{q}_{2}=b_{1}+b_{2} \cos (S), \mathrm{q}_{3}=0,
$$

the growth rate at each point remains the same while the generating curve expands. Hence, there will be a steeper growth gradient along the growth axis when the generating curve is smaller, and the surface will rotate more tightly as compared to a larger generating curve. For this form of growth, the cell tracks trace out a logarithmic spiral, and a surface similar to the shell of a Nautilus or Ammonite is created (Figure 4a). If we take instead

$$
\mathrm{q}_{1}=-c, \mathrm{q}_{2}=\lambda\left(b_{1}+b_{2} \cos (S)\right), \mathrm{q}_{3}=0,
$$

where we have scaled the binormal growth with the stretch factor $\lambda=\lambda(t) \equiv$ $1+c t$, the growth rate in the binormal direction scales with size, and the gradient of the linear growth profile remains fixed. Here, the cell tracks trace out an algebraic spiral; an example of the resulting structure appears in Figure 4b. Now, consider

$$
\mathrm{q}_{1}=-c, \mathrm{q}_{2}=b_{1}+b_{2} \lambda \cos (S), \mathrm{q}_{3}=0 .
$$

Here, the growth gradient is fixed, independent of the size of the generating curve. Hence, the tightness of the rotation is independent of size, and the center of the generating curve traces out a circle, producing a structure as shown in Figure 4c.

\subsection{On self-similarity in space and time}

A remarkable feature of certain seashells is the property of self-similarity. However, there are some intricacies in defining the notion of self-similarity. We think of a structure as being self-similar if it is scale invariant, i.e. if the structure continued infinitely we should see the same structure no matter how far we zoomed in or out. This is most readily thought of as a global property; for instance we could define the requirement that the ratio of distances between successive points be fixed with each complete $2 \pi$ turn, but this requires a global definition of successive points. How do we define self-similarity at a local level? In view of Figure 4, we recognize that (a) is the only self-similar structure. This is easily verified by computing the ratio of distances between successive intersections with the $x$-axis, or by observing that the the path of any material point follows a logarithmic spiral, a well-known self-similar structure. The key feature for a local definition of self-similarity is the observation that the growth vector at any given point on the generating curve should be invariant up to a scale. This can be directly observed from Equation (27), where the ratio $\mathrm{q}_{1} / \mathrm{q}_{2}$ is fixed for each point $S$, which is not the case for (b) or $(\mathrm{c})$. 
a)

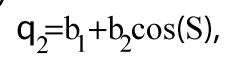
$\mathrm{q}_{1}=-c, \mathrm{q}_{3}=0$
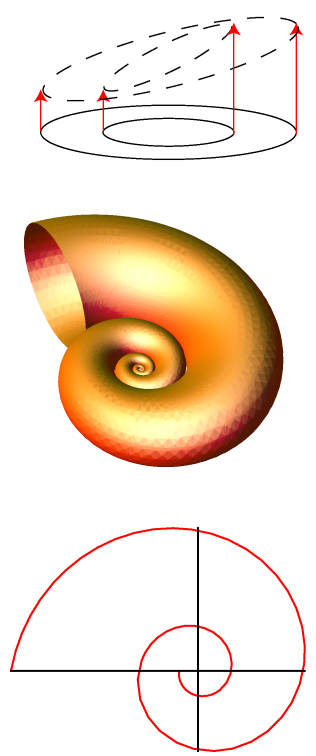

b)

$$
\begin{aligned}
& \mathrm{q}_{2}=\lambda\left(\mathrm{b}_{1}+\mathrm{b}_{2} \cos (\mathrm{S})\right), \\
& \mathrm{q}_{1}=-c, \mathrm{q}_{3}=0
\end{aligned}
$$
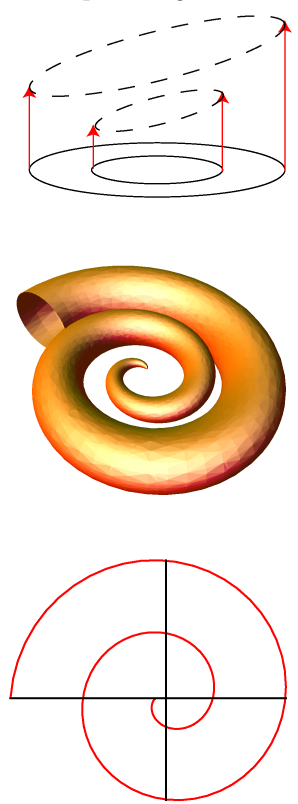

c)

$$
\begin{aligned}
& \mathrm{q}_{2}=\mathrm{b}_{1}+\mathrm{b}_{2} \lambda \cos (\mathrm{S}), \\
& \mathrm{q}_{1}=-c, \mathrm{q}_{3}=0
\end{aligned}
$$
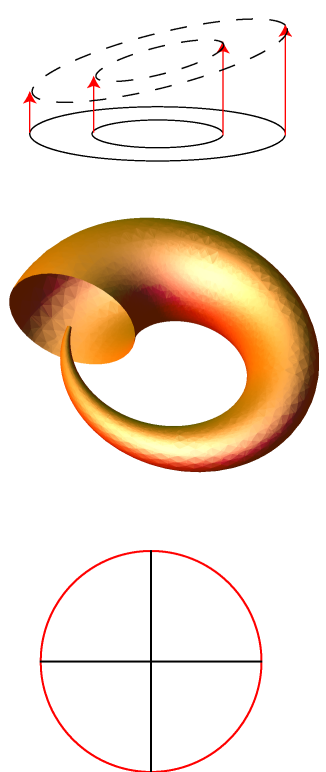

Fig. 4 Three primary forms of growth with a linearly expanding radius. For each case, we show from top to bottom: the local velocity, a schematic illustrating the binormal growth gradient as the radius changes, a sample structure, and the curve traced out by a fixed material point.

Explicitly missing from this notion of self-similarity, though, is time. That is, the final structure in Fig. 4a is self-similar, but we still might not wish to call this self-similar growth, because the structure does not grow invariantly in time. Consider alternatively the growth law

$$
\mathrm{q}_{1}=-\lambda c, \mathrm{q}_{2}=\lambda\left(b_{1}+b_{2} \cos (S)\right), \mathrm{q}_{3}=0 .
$$

The only difference is that each velocity component is scaled by the stretch factor $\lambda$. The direction of the growth vector is still time independent (i.e. ratio $\mathrm{q}_{1} / \mathrm{q}_{2}$ is fixed), and in fact this growth produces an identical structure to Fig. 4a. The difference is that for the growth of Equations (30), the ratio of the magnitude of the growth vector to the length scale of the generating curve (radius or size) is also independent of time. Thus, this growth is fully self-similar. This is pictured schematically in Figure 5 .

\section{Application to seashell growth}

As noted, Equations (27) and (30) produce identical structures, and one must look at time as an explicit parameter to see the difference. With regards to 
seashell models, the inclusion of time as an explicit parameter is a fairly recent development (Rice 1998), and is of great importance in understanding the growth process (Urdy et al 2010). The growth governed by Equations (27) is not time invariant and so the coiling rate - the time it takes to coil around one time, or to complete one whorl in the terminology of seashells - is not fixed; rather the time to coil around increases exponentially while the aperture size increases linearly. For the growth governed by Equations (30), on the other hand, the time to complete one coil is fixed, independent of size or time. In order to do this, though, material deposition rates must increase exponentially (note that the radius is an exponential function of time). As exponentially increasing growth rates are not very realistic, this would seem to preclude the possibility of time invariant growth. In fact, in the case of seashells, several empirical studies of molluscs (Black et al 1994; Iijima 2001; Schöne et al 2007) have found that in fact shell growth rates decrease with increasing octogenetic age.

Perhaps the most natural form of growth is one for which the rate of increase in shell surface area is constant. To see how this is translated into local growth velocities, we define the material rate $\dot{m}$ as the rate of increase of surface area. We can compute $\dot{m}$ for a given time $t$ by integrating the magnitude of the velocity field over the generating curve,

$$
\dot{m}(t)=\int_{\mathcal{C}}\left|\mathbf{q}_{1} \mathbf{d}_{1}+\mathrm{q}_{2} \mathbf{d}_{2}+\mathrm{q}_{3} \mathbf{d}_{3}\right| d s=\int_{\mathcal{C}}\left(\mathrm{q}_{1}^{2}+\mathrm{q}_{2}^{2}+\mathrm{q}_{3}^{2}\right)^{1 / 2} d s,
$$

where $\mathcal{C}$ denotes the generating curve at time $t$. Here $s$ is the arc length of the generating curve at time $t$, which relates to the material parameter $S$ by $s=\frac{\lambda}{\lambda_{0}} S$. For a circular generating curve with $\lambda_{0}=1$, we have

$$
\dot{m}=\int_{0}^{2 \pi}\left(\mathrm{q}_{1}^{2}+\mathrm{q}_{2}^{2}+\mathrm{q}_{3}^{2}\right)^{1 / 2} \lambda d S
$$

To have a constant material rate, $\dot{m}$ must be independent of time. Since $\lambda=$ $\lambda(t)$, this is accomplished by scaling each local velocity $\mathbf{q}_{i}$ with $\lambda^{-1}$. Thus, for the self-similar shape under consideration, we find that the material rate is independent of time if the local growth is given by

$$
\mathrm{q}_{1}=-\frac{c}{\lambda}, \quad \mathrm{q}_{2}=\frac{b_{1}+b_{2} \cos (S)}{\lambda}, \mathrm{q}_{3}=0
$$

This form also produces a self-similar shape in space, but here the rate of deposition scales inversely with the size of the aperture (see Figure 5). Also, the coiling rate is a logarithmic function of time, while the radius increases as $t^{1 / 2}$. This form is probably the most realistic in terms of shell growth ${ }^{2}$, and followed naturally from the assumption of a constant material rate.

${ }^{2}$ For a detailed discussion on time and growth rates in seashells, see (Urdy et al 2010). 
(a)

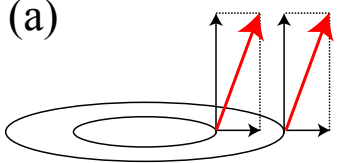

$\mathrm{q}_{2}=b_{1}+b_{2} \cos (S)$

$\mathrm{q}_{1}=-c, \quad \mathrm{q}_{3}=0$ (b)

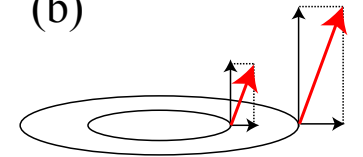

$\mathrm{q}_{2}=\lambda\left(b_{1}+b_{2} \cos (S)\right)$

$\mathrm{q}_{1}=-\lambda c, \quad \mathrm{q}_{3}=0$ (c)

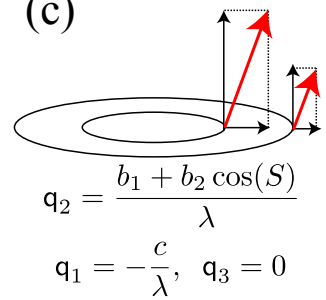

Fig. 5 Growth vectors for a fixed point on the generating curve and two different aperture sizes for the growth of (a): Equations (27), growth with constant deposition rate; (b): Equations (30), fully self-similar growth; and (c): Equations (33), growth with a constant material rate, i.e. rate of increase of surface area .

\subsection{Out of plane growth}

In the previous examples, each point of the generating curve has followed a path in time that has been either linear or planar. For more complex structures, we need to consider non-planar growth. For simplicity, we keep a circular generating curve. There are several forms of local velocity fields of interest that produce non-planar growth. We will present three different forms that produce similar (and even identical) surfaces, yet have distinct and important differences from a biological viewpoint.

I. Global velocity component: One way to obtain non-planar growth is to add a global velocity component of the form $\mathbf{q}_{g}=\mathbf{q}_{g}(t)$, that is for given time, we impose the same velocity at every point on the generating curve. This is equivalent to imposing a rigid translation of the generating curve. Computationally, the key is to express the global velocity in the local components. In general, to add the global velocity component $\mathbf{q}_{g}$, we decompose

$$
\mathbf{q}_{g}=\alpha_{1} \mathbf{d}_{1}+\alpha_{2} \mathbf{d}_{2}+\alpha_{3} \mathbf{d}_{3},
$$

where $\alpha_{i}=\mathbf{q}_{g} \cdot \mathbf{d}_{i}$. In the case of Examples 3.3-3.4, the curve corresponding to the point $S=0$ lives in the $x$ - $z$ plane. Adding the velocity $\mathbf{q}_{g}=k \mathbf{e}_{y}$ adds a uniform component in the $y$-direction. A global velocity is trivial to add from an analytical and computational standpoint, as it has no effect on the local orientation of the frames. To see this, note that $\mathbf{q}_{g}$ can be expressed via (34) as local velocity $\mathbf{q}_{g}=\mathbf{D}^{T} \cdot \mathbf{q}_{g}$. It is straightforward to show that $\mathbf{q}_{g}^{\prime}=-\mathbf{U} \cdot \mathbf{q}_{g}$, and hence the velocity terms cancel out of the compatibility equations (see Equation (11)).

II. Rotating growth axis: Another way for non-planar growth to occur is for the growth axis to rotate in time. For the binormal velocity $\mathrm{q}_{2}=b_{1}+b_{2} \cos (S)$, the growth axis is always on a line contained in the $x-z$ plane, and thus the rotation remains in this plane. If this growth axis locally rotates during the 
evolution, the direction of rotation will also vary and growth will be nonplanar. To illustrate, consider the velocity field

$$
\mathrm{q}_{1}=-c, \quad \mathrm{q}_{2}=b_{1}+b_{2} \cos \left(S-b_{3} t\right), \quad \mathrm{q}_{3}=0 .
$$

Here the maximal growth (if $b_{2}>0$ ) occurs at material point $S=b_{3} t$, thus the parameter $b_{3}$ dictates the rotation rate of the growth axis.

III. Tangential velocity: An alternative form to rotating the growth axis is to add a tangential velocity component. Consider the velocity field

$$
\mathrm{q}_{1}=-c, \mathrm{q}_{2}=b_{1}+b_{2} \cos (S), \quad \mathrm{q}_{3}=b_{3} .
$$

The tangential component effectively rotates the growth axis with respect to a global coordinate system, and this field produces an identical surface to (35). There is, however, a distinction between the two, which although subtle, is biologically relevant. The difference is understood in terms of cell tracks. In (36), maximal growth always occurs at a fixed material point (the point $S=0$ if $b_{2}>0$ ), and this point moves in the tangential direction with time, hence rotating the growth axis around the generating curve. Essentially, each material point has a fixed growth rate and non-planar growth occurs due to tangential motion along the generating curve. In (35), there is no tangential motion and material points stay in the same local location on the generating curve, but the growth rate for any given material point on the generating curve varies with time and hence the location where growth is maximal also varies.

This idea is illustrated in Figure 6, in which surfaces are plotted using a rotating growth axis and a tangential velocity component (the forms are slightly different from Equations (35) and (36); the scale factor $\lambda$ has been included in the velocities for computational ease, but the idea is the same). The same values of $c, b_{1}, b_{2}$, and $b_{3}$ were used in each case, and the resulting surfaces are identical. Included on each surface is a red curve representing a cell track for the particular value $S=0$. In the case of the rotating growth axis, the binormal velocity of the cell varies with time, and the cell track oscillates between the inner and outer edge of the global structure. For the tangential velocity case, on the other hand, $S=0$ always corresponds to the point of maximal growth, and the cell track follows the outer edge of the global structure.

Given the structure for out-of-plane growth and rotation we have developed, by varying the parameters and/or letting the rotation rates vary in time, we can generate several surfaces of interest in the study of seashells and horns. The mathematical foundation leads to a simplicity in form: surfaces can be generated with a minimal number of parameters, and an analytical solution can be obtained, i.e. the frame vectors $\mathbf{d}_{i}(\sigma, t)$ and the parameterised surface $\mathbf{r}(\sigma, t)$ can be written explicitly. Figure 7 displays three surfaces, the form of velocity field that produced them, and a related biological entity. A global velocity was added for the out-of-plane component of the growth. Similar structures could be obtained with a rotating growth axis or a tangential velocity, but finding an explicit solution is more challenging. 
a) Rotating growth axis $\mathrm{q}_{1}=-c$, $\mathrm{q}_{2}=\lambda\left(\mathrm{b}_{1}+\mathrm{b}_{2} \cos \left(\mathrm{S}-\mathrm{b}_{3} \mathrm{t}\right)\right)$ $\mathrm{q}_{3}=0$ b) Tangential velocity component $\mathrm{q}_{1}=-c$, $\mathrm{q}_{2}=\lambda\left(\mathrm{b}_{1}+\mathrm{b}_{2} \cos (\mathrm{S})\right.$ $\mathrm{q}_{3}=\lambda \mathrm{b}_{3}$

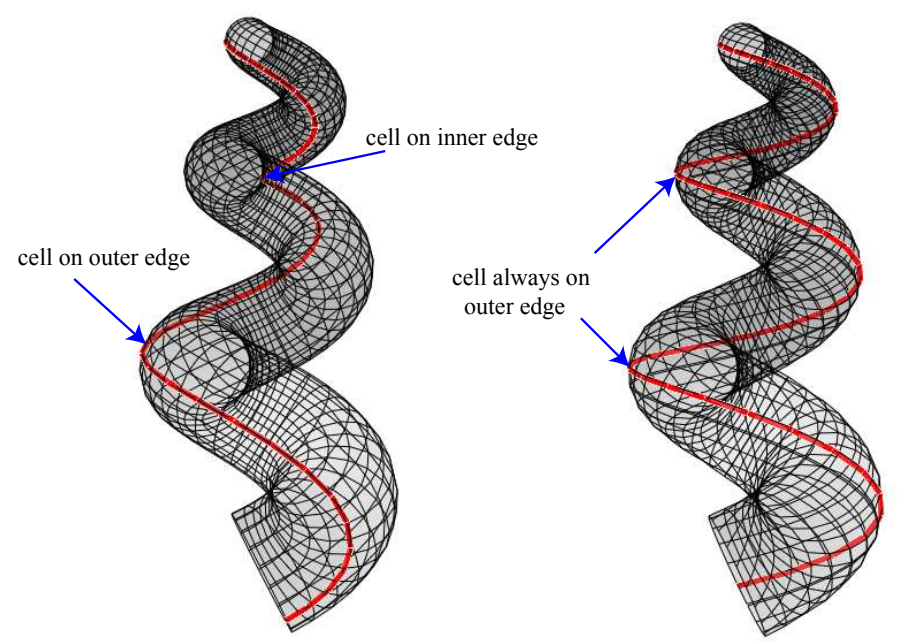

Fig. 6 Comparison of two primary forms of local velocity for out-of-plane growth. The plotted surfaces are identical, but with different parameterisations, reflecting the distinct difference in cell tracks. The parameters are $c=0.12, b_{1}=1.6, b_{2}=1, b_{3}=0.55$ for each surface.

\section{Arbitrary generating curves and extrinsic construction}

Through the previous examples, we have built up relationships and rules on the form of local growth velocity to generate a number of structures with a circular cross-section. In this section, we consider an arbitrary generating curve. We then describer a different approach to surface construction, one which has been utilised in previous shell growth simulations, and show the connection to our approach.

\subsection{Shape constancy}

The primary reason that the system is tractable in the examples we have considered is that the generating curve remains a circle. This points to a general class of evolutions which are in principal analytically tractable, that is if the shape of the generating curve does not change. From a global perspective, this means that the curve can only undergo translation, rotation, and dilation. How does this translate to the form of the local velocity? To answer this, we restrict to planar curves and note the distinction between in-plane curve 
a) $\mathrm{q}_{1}=-(c+k \sin (\mathrm{S}))$,

$\mathrm{q}_{2}=\mathrm{b}_{1}+\mathrm{b}_{2} \cos (\mathrm{S})$

$\mathrm{q}_{3}=k \cos (\mathrm{S})$
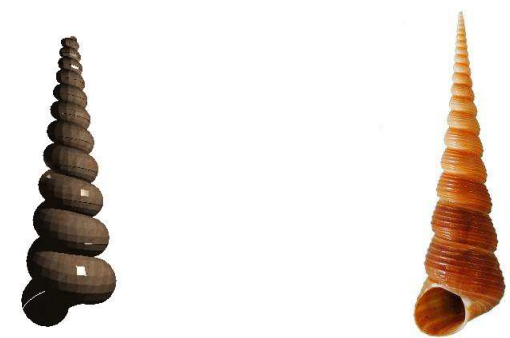

Turitella seashell

b) $\mathrm{q}_{1}=-(c+k(\mathrm{t}) \sin (\mathrm{S}))$, $q_{2}=b_{1}+\lambda b_{2} \cos (S)$ $\mathrm{q}_{3}=k(\mathrm{t}) \cos (\mathrm{S})$, with $k=k_{1}+k_{2}$ t

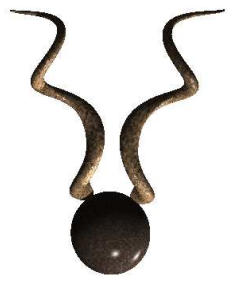

c) $\mathrm{q}_{2}=\mathrm{b}_{1}+\mathrm{b}_{2}(\mathrm{t}) \cos (\mathrm{S})$ with $b_{2}$ of form:

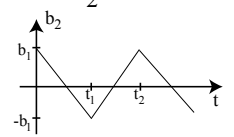

plus global velocity: $\mathrm{q}_{\mathrm{g}}=\left\{\begin{array}{l}k_{1} \mathbf{e}_{\mathrm{y}}: \mathrm{t}<\mathrm{t}_{1} \\ k_{2} \mathbf{e}_{\mathrm{x}}: \mathrm{t}_{1}<\mathrm{t}<\mathrm{t}_{2} \\ -k_{3} \mathbf{e}_{\mathrm{y}}:\end{array}\right.$
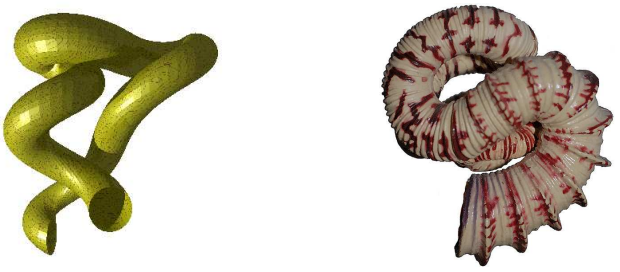

Nipponite shell

Fig. 7 Examples of surfaces similar to biological structures: a Turitella seashell (a), Antelope horns (b), and a Nipponite shell (c). The form of the velocity field and a sample structure are shown. In each case, an explicit solution for the curve evolution can be obtained. Parameters: for (a), $b_{1}=1, b_{2}=10, c=-0.025, k=0.32$, and initial radius 0.1 ; for (b), $b_{1}=0.75, b_{2}=0.3, c=-0.04, k_{1}=0.25, k_{2}=0.02$, and initial radius 0.1 ; for (c), $t_{1}=12, t_{2}=20.4, k_{1}=0.8, k_{2}=0.73, k_{3}=1.2$, and radius 1 .

evolution, due to velocity in the normal and tangential directions, and out-ofplane evolution, due to velocity in the binormal direction. We consider each separately.

\subsubsection{In-plane velocity}

Consider an arbitrary planar curve $\mathbf{r}(S, t)$. Suppose without loss of generality that the curve is initially parameterised by arc length, so that $\left|\mathbf{r}^{\prime}(S, 0)\right| \equiv \lambda_{0}=$ 1. We wish to find the form of $q_{1}$ and $q_{3}$ so that the shape of the curve does not change as the curve evolves. Shape constancy implies that at any time 
$t,\left|\mathbf{r}^{\prime}\right|=\lambda=\lambda(t)$, and that the curvature $\kappa(S, t)$ is just the initial curvature scaled by the dilation factor $\lambda$,

$$
\kappa(S, t)=\lambda^{-1} \kappa(S, 0) .
$$

Working in the Frenet frame, the shape variables are then

$$
\mathrm{u}_{1}=\mathrm{u}_{3}=0, \quad \mathbf{u}_{2}=\lambda \kappa=\kappa(S, 0) .
$$

This implies that $\dot{\mathrm{u}}_{2}=0$, a key characteristic of shape invariance. Setting $\dot{\mathrm{u}}_{2}=0$ in the compatibility equations (13), (15), (17), we have

$$
\begin{aligned}
& \mathrm{q}_{1}^{\prime}+\mathrm{u}_{2} \mathrm{q}_{3}=\lambda \mathrm{w}_{2} \\
& \mathrm{q}_{3}^{\prime}-\mathrm{u}_{2} \mathrm{q}_{1}=\dot{\lambda} \\
& \mathrm{w}_{2}^{\prime}=0 .
\end{aligned}
$$

Taking a spatial derivative across (39) and combining with (40), (41), we obtain

$$
\mathrm{q}_{1}^{\prime \prime}+\mathrm{u}_{2} \mathrm{q}_{3}+\mathrm{u}_{2}^{2} \mathrm{q}_{1}+\mathrm{u}_{2} \dot{\lambda}-\lambda^{\prime} \mathrm{w}_{2}=0 .
$$

This relationship provides a rule on the allowable form of the local in-plane velocity fields in order for the shape of the generating curve to remain constant. We next construct the general solution to (42). In global coordinates, the solution is of the form

$$
\mathbf{q}=c_{1}(t) \mathbf{r}+c_{2}(t) \mathbf{r}_{\perp}+\mathbf{a}(t) .
$$

The first term describes a velocity in the radial direction and accounts for dilation of the curve. The second term gives a velocity in the circumferential direction and accounts for rotation $\left(\mathbf{r}_{\perp}\right.$ is a vector perpendicular to the position vector $\mathbf{r}$ and with the same magnitude). In the third term, $\mathbf{a}(t)$ is an arbitrary vector; this term provides a rigid translation. To see that the form (43) satisfies (42) and to determine the explicit form of the $c_{i}$, we express $\mathbf{q}$ in the local coordinates. This is achieved by writing

$$
\mathbf{r}=\beta_{1} \mathbf{d}_{1}+\beta_{3} \mathbf{d}_{3}, \quad \mathbf{r}_{\perp}=\beta_{3} \mathbf{d}_{1}-\beta_{1} \mathbf{d}_{3}, \quad \mathbf{a}=\alpha_{1} \mathbf{d}_{1}+\alpha_{3} \mathbf{d}_{3},
$$

where $\beta_{i}=\mathbf{r} \cdot \mathbf{d}_{i}, \alpha_{i}=\mathbf{a} \cdot \mathbf{d}_{i}$. The local velocities are then

$$
\mathbf{q}_{1}=c_{1} \beta_{1}+c_{2} \beta_{3}+\alpha_{1}, \quad \mathbf{q}_{3}=c_{1} \beta_{3}-c_{2} \beta_{1}+\alpha_{3} .
$$

Recalling that $\mathbf{r}^{\prime}=\lambda \mathbf{d}_{3}$ and the Frenet relations $\mathbf{d}_{1}^{\prime}=-\mathrm{u}_{2} \mathbf{d}_{3}, \mathbf{d}_{3}^{\prime}=\mathrm{u}_{2} \mathbf{d}_{1}$, we have

$$
\begin{aligned}
& \beta_{1}^{\prime}=-\mathrm{u}_{2} \beta_{3}, \quad \beta_{3}^{\prime}=\lambda+\mathrm{u}_{2} \beta_{1} \\
& \alpha_{1}^{\prime}=-\mathrm{u}_{2} \alpha_{3}, \quad \alpha_{3}^{\prime}=\mathrm{u}_{2} \alpha_{1} .
\end{aligned}
$$

Then, inserting (45) into (42), we find that the relation is satisfied if

$$
c_{1}(t)=\frac{\dot{\lambda}}{\lambda}, \quad c_{2}(t)=w_{2}(t),
$$

which highlights the relationship between the dilation factors $c_{1}$ and $\dot{\lambda}$ as well as the equivalence between the rotation factor $c_{2}$ and the frame rotation component $\mathrm{w}_{2}$. 

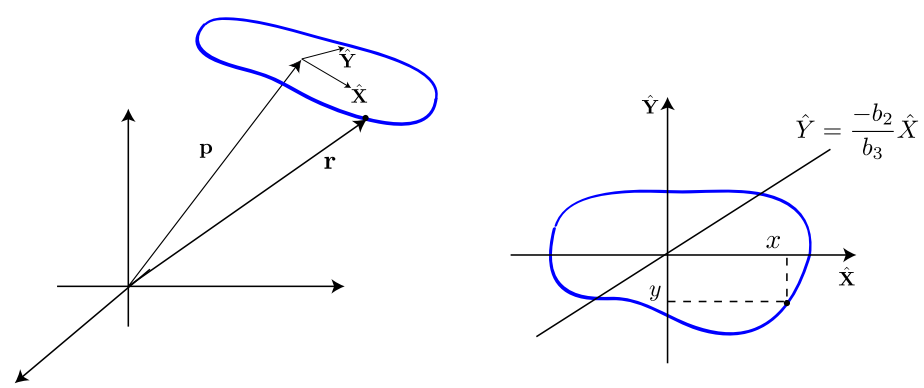

Fig. 8 A planar curve is represented by a position vector $\mathbf{p}$ and the unit vectors $\hat{\mathbf{X}}$ and $\hat{\mathbf{Y}}$, which form a basis for the plane of the curve.

\subsubsection{Binormal velocity}

In the previous subsection we determined the form of local in-plane velocities to keep shape constancy. Here we consider a similar problem for binormal, outof-plane velocity. First, it is instructive to consider the more general constraint that the curve merely remains planar. That is, we wish to find the form of binormal velocity so that the curve remains planar, but we allow the in-plane shape of the curve to evolve arbitrarily. Let $\mathbf{r}(S, t)$ be a planar curve. Planarity implies that the curve can be expressed as

$$
\mathbf{r}(S, t)=\mathbf{p}(t)+x(S, t) \hat{\mathbf{X}}+y(S, t) \hat{\mathbf{Y}}
$$

where $\mathbf{p}$ is a position vector to a point in the plane of the curve and the unit vectors $\hat{\mathbf{X}}, \hat{\mathbf{Y}}$ form a basis for the plane of the curve. This is illustrated in Figure 8. In this representation, the quantities $\lambda$ and $\kappa$ take the form

$$
\lambda=\sqrt{x^{\prime 2}+y^{\prime 2}}, \quad \kappa=\frac{x^{\prime} y^{\prime \prime}-x^{\prime \prime} y^{\prime}}{\left(x^{\prime 2}+y^{\prime 2}\right)^{3 / 2}} .
$$

In order for the curve to remain planar, it must hold that the torsion vanish, i.e. $\mathrm{u}_{3}=\dot{\mathrm{u}}_{3}=0$ (recall $\mathrm{u}_{1}=0$ by definition for the Frenet frame). Inserting this in Equations (14), (16), (18), we have

$$
\begin{aligned}
& \mathrm{q}_{2}^{\prime}=-\lambda \mathrm{w}_{1} \\
& \mathrm{w}_{1}^{\prime}=-\mathrm{u}_{2} \mathrm{w}_{3} \\
& \mathrm{w}_{3}^{\prime}=\mathrm{u}_{2} \mathrm{w}_{1} .
\end{aligned}
$$

Taking a derivative across (52) and using (51), (53), we obtain the following ODE for $\mathrm{q}_{2}$ :

$$
\mathrm{q}_{2}^{\prime \prime \prime}-\mathrm{q}_{2}^{\prime \prime}\left(\frac{2 \lambda}{\lambda}+\frac{\mathrm{u}_{2}^{\prime}}{\mathrm{u}_{2}}\right)+\mathrm{q}_{2}^{\prime}\left(\mathrm{u}_{2}^{2}+\frac{2 \lambda^{\prime 2}}{\lambda^{2}}-\frac{\lambda^{\prime \prime}}{\lambda}+\frac{\mathrm{u}_{2}^{\prime} \lambda^{\prime}}{\mathrm{u}_{2} \lambda}\right)=0
$$


The general solution of which is

$$
\mathrm{q}_{2}=b_{1}(t)+b_{2}(t) x(S, t)+b_{3}(t) y(S, t) \text {, }
$$

which is easily found to be true by direct substitution and use of the formulas (50). Note that if the shape of the generating curve remains fixed, the functions $x$ and $y$ are scalar multiples of the initial curve $\mathbf{r}(S, 0)=\left[x_{0}(S), y_{0}(S), 0\right]$. The form (55) implies a remarkable universal feature of growth. Namely if the generating curve remains planar, then there exists a distinguished axis along the vector $b_{2} \hat{\mathbf{X}}+b_{3} \hat{\mathbf{Y}}$ such that the binormal growth on the generating curve is a linear function when projected onto this axis. This is exactly what we defined as the growth axis in the case of a circular generating curve. In particular, for the initial curve $\mathbf{r}(S, 0)=[\cos (S), \sin (S), 0]$, the form is $\mathrm{q}_{2}=b_{1}+b_{2} \cos (S)+b_{3} \sin (S)$. Taking $b_{3}=0$ fixes the growth axis as the $x$ axis of the initial plane, and we recover the growth law for the torus, Equation (24).

\section{Application to seashell growth}

The fact that the binormal component of growth must be linear along an axis highlights the notion of a growth gradient. The idea of a growth gradient has been utilised in studies of shell growth for circular generating curves previously, e.g. (Rice 1998), and the general property has been hinted at in (Hammer and Bucher 2005), but without mathematical analysis. In consideration of shell growth from a biological perspective, it is a fascinating property. How does the shell "recognise" the growth axis? After all, the growth is not a linear function along the aperture itself but rather along an axis that is separate from the shell altogether. One might argue that the rate of growth at each point, and hence the growth axis, is genetically coded. However, this is not entirely satisfying, especially when considering that the rule holds even if the shape of the curve evolves arbitrarily. Such a scenario is of relevance, as numerous examples of shells can be found in which the shape of the aperture remains planar but changes with time while the shell coiling continues with great regularity. For instance, in many shells in the Murex genus, the aperture undergoes dramatic shape change during the formation of ornamentation. At each step, the cell growth rates must be updated for the new locations along the axis. How the mollusc accomplishes this remains an intriguing mystery.

\subsubsection{Bivalve shell}

In the previous subsections we have outlined the rules on local velocity to evolve an arbitrary planar curve while maintaining the same shape. As an example of a surface with non-circular cross section, we now grow a bivalve shell. The initial curve, defining the cross section, is given by $\mathbf{r}_{0}=[R \cos (S), R \sin (S), 0]$, with $R=1+0.05 \cos (10 S)$, and the local growth law is composed of binormal growth linear along a fixed axis (see Figure 9) with a time varying coiling rate, 


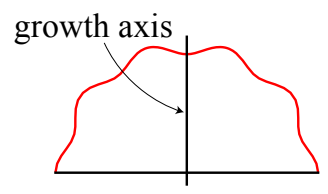

cross section
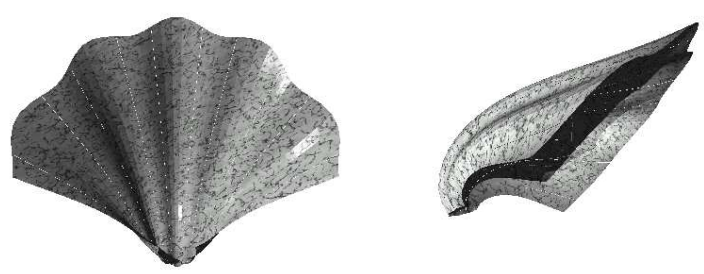

Fig. 9 Growth of a bivalve shell, using the cross section shown at left and velocity described in the text, composed of rotating and dilation.

as well as a fixed dilation component. Explicitly, the velocities are

$$
\begin{aligned}
& \mathbf{q}_{1}=-c\left(\mathbf{r}_{0} \cdot \mathbf{d}_{1}(S, 0)\right) \\
& \mathrm{q}_{2}=b_{1}+b_{2}(t) R \cos (S) \\
& \mathbf{q}_{3}=-c\left(\mathbf{r}_{0} \cdot \mathbf{d}_{3}(S, 0)\right),
\end{aligned}
$$

where $c=3, b_{1}=0.1$, and the rotation rate is a linear function of time, $b_{2}=5-0.5 t$. The result is shown in Figure 9. Even for this structure, an explicit solution to the curve evolution can be obtained.

\subsection{Connection with extrinsic construction}

In this section, we consider an extrinsic surface construction and then work backwards to relate to a local velocity field. The idea is to begin with a centerline curve, equipped with a local frame, and "dress" it with a shape, a "dressing curve," at every point to create a surface. For instance, one could "dress" a straight line with a circle to create a cylinder. Essentially, this is the moving frame approach introduced by Okamoto (Okamoto 1988a) for seashells; notice that while it can provide a convenient way to construct surfaces, it is somewhat disconnected from the growth process. Our goal is to formulate this idea generally and connect it to the intrinsic description presented so far. Formally, we consider an arbitrary curve $\mathbf{x}(t)$, attached with a local right orthonormal frame $\hat{\mathbf{d}}_{i}, i=1,2,3$, such that $\hat{\mathbf{d}}_{3}$ is tangent to $\mathbf{x}$ (although this condition could be relaxed). Assume without loss of generality that $\mathbf{x}(t)$ is parameterised by arc length $t$. We also make the assumption that the dressing curve lives in a plane perpendicular to $\hat{\mathbf{d}}_{3}$, and thus identify $\hat{\mathbf{d}}_{1}$ and $\hat{\mathbf{d}}_{2}$ as director vectors for the dressing curve. At any time $t$, the dressing curve is given by an arbitrary shape $\mathcal{S}$, defined in terms of the director vectors as:

$$
\rho(S, t)=r_{1}(S) \hat{\mathbf{d}}_{1}(t)+r_{2}(S) \hat{\mathbf{d}}_{2}(t),
$$

along with dilation factor $\lambda(t)$, so that the surface is defined by

$$
\mathbf{r}(S, t)=\mathbf{x}(t)+\lambda(t) \rho(S, t) .
$$




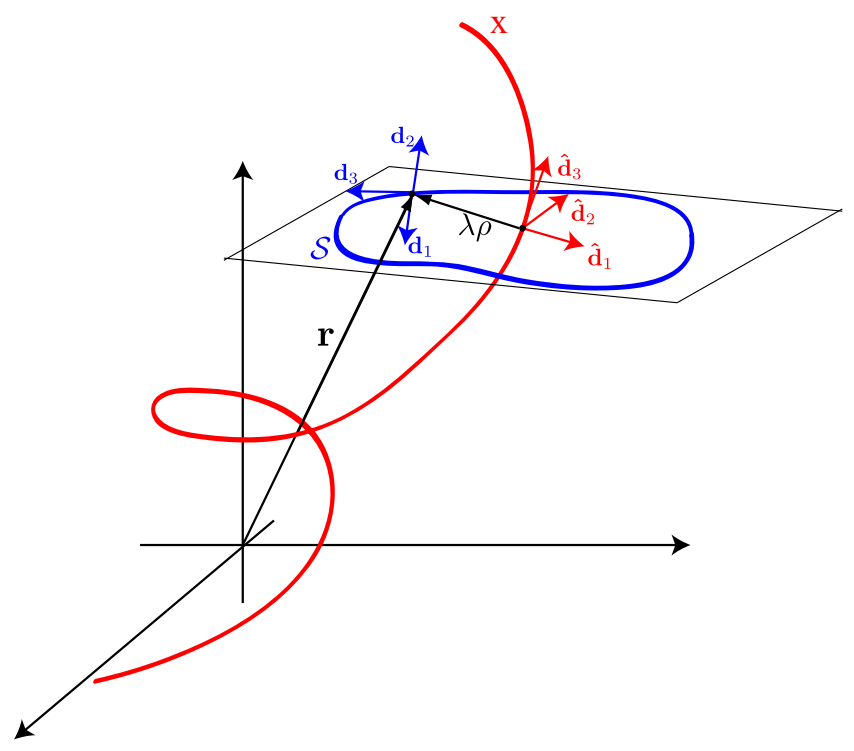

Fig. 10 Setup for the "curve dressing" approach. The shape $\mathcal{S}$ lives in the plane of the director vectors $\hat{\mathbf{d}}_{1}$ and $\hat{\mathbf{d}}_{2}$, the vectors $\mathbf{d}_{i}, i=1,2,3$ form a basis for the curve defining $\mathcal{S}$.

This is illustrated in Figure 10. This is a convenient way to build surfaces, and this basic idea has been utilised in previous studies (Fowler and Meinhardt 1992; Okamoto 1988a; van der Helm et al 1998). With an appropriate choice of $\rho$ and $\mathbf{x}$ the surfaces we have described in this paper can be created within this framework. To connect to the intrinsic method of the previous sections, we associate the shape $\mathcal{S}$ of this extrinsic description with the generating curve of the intrinsic description. For the surface (58), we can determine the local velocity field as

$$
\partial_{t} \mathbf{r}=\mathrm{q}_{1} \mathbf{d}_{1}+\mathrm{q}_{2} \mathbf{d}_{2}+\mathrm{q}_{3} \mathbf{d}_{3},
$$

where $\mathbf{d}_{i}, i=1,2,3$ form a local basis for the generating curve $\mathcal{S}$. Having chosen a basis, the local velocity components are

$$
\mathrm{q}_{i}=\partial_{t} \mathbf{r} \cdot \mathbf{d}_{i} .
$$

We use the Frenet frame for $\mathcal{S}$, so that

$$
\mathbf{d}_{3}=\frac{\partial_{s} \mathbf{r}}{\left|\partial_{s} \mathbf{r}\right|}, \quad \mathbf{d}_{1}=\frac{\partial_{s} \mathbf{d}_{3}}{\left|\partial_{s} \mathbf{d}_{3}\right|}, \quad \mathbf{d}_{2}=\mathbf{d}_{3} \times \mathbf{d}_{1} .
$$

It should be clarified that the $\hat{\mathbf{d}}_{i}$ form a local basis for the centerline curve, while the $\mathbf{d}_{i}$ are the basis for the shape $\mathcal{S}$, i.e. the familiar basis vectors for the generating curve (see Figure 10). In this approach, the form of the local velocity is determined by the choice of frame for the centerline curve, i.e. the 
$\hat{\mathbf{d}}_{i}$. We consider two cases.

I. Frenet frame: If the Frenet frame is chosen for the $\hat{\mathbf{d}}_{i}$, then

$$
\begin{aligned}
& \partial_{t} \mathbf{x}=\hat{\mathbf{d}}_{3}^{F} \\
& \partial_{t} \hat{\mathbf{d}}_{1}^{F}=-\kappa \hat{\mathbf{d}}_{3}^{F}+\tau \hat{\mathbf{d}}_{2}^{F} \\
& \partial_{t} \hat{\mathbf{d}}_{2}^{F}=-\tau \hat{\mathbf{d}}_{1}^{F} \\
& \partial_{t} \hat{\mathbf{d}}_{3}^{F}=\kappa \hat{\mathbf{d}}_{1}^{F},
\end{aligned}
$$

where we use the superscript $F$ to denote the Frenet frame. Taking a $t$ derivative of (58) and using (62)-(65) to simplify the components (60), we obtain

$$
\begin{aligned}
& \mathrm{q}_{1}^{F}=\dot{\lambda} \frac{r_{1} r_{1}^{\prime \prime}+r_{2} r_{2}^{\prime \prime}}{\gamma}+\lambda \tau \frac{r_{1} r_{2}^{\prime \prime}-r_{2} r_{1}^{\prime \prime}}{\gamma} \\
& \mathbf{q}_{2}^{F}=\left(1-\lambda \kappa r_{1}\right) \frac{r_{1}^{\prime} r_{2}^{\prime \prime}-r_{2}^{\prime} r_{1}^{\prime \prime}}{\gamma} \\
& \mathbf{q}_{3}^{F}=\dot{\lambda}\left(r_{1} r_{1}^{\prime}+r_{2} r_{2}^{\prime}\right)+\lambda \tau\left(r_{1} r_{2}^{\prime}-r_{2} r_{1}^{\prime}\right),
\end{aligned}
$$

where $\gamma=\left(r_{1}^{\prime \prime 2}+r_{2}^{\prime \prime 2}\right)^{1 / 2}$. A key observation is that the shape of the centerline, captured by the curvature $\kappa$ and torsion $\tau$, is distributed across all three velocity components.

II. Natural frame: Suppose instead that the natural frame is chosen for the $\hat{\mathbf{d}}_{i}$ (Bishop 1975), defined so that there is no twist about the tangent. The basis satisfies:

$$
\begin{aligned}
& \partial_{t} \mathbf{x}=\hat{\mathbf{d}}_{3}^{N} \\
& \partial_{t} \hat{\mathbf{d}}_{1}^{N}=-k_{1} \hat{\mathbf{d}}_{3}^{N} \\
& \partial_{t} \hat{\mathbf{d}}_{2}^{N}=-k_{2} \hat{\mathbf{d}}_{3}^{N} \\
& \partial_{t} \hat{\mathbf{d}}_{3}^{N}=k_{1} \hat{\mathbf{d}}_{1}^{N}+k_{2} \hat{\mathbf{d}}_{2}^{N},
\end{aligned}
$$

where superscript $N$ denotes the natural frame and $k_{1}$ and $k_{2}$ satisfy

$$
\theta=\arctan \frac{k_{2}}{k_{1}}, \quad \kappa=\left(k_{1}^{2}+k_{2}^{2}\right)^{1 / 2}, \text { where } \theta^{\prime}(t)=\tau(t) .
$$

In this case, the components (60) may be simplified to:

$$
\begin{aligned}
& \mathrm{q}_{1}^{N}=\dot{\lambda} \frac{r_{1} r_{1}^{\prime \prime}+r_{2} r_{2}^{\prime \prime}}{\gamma} \\
& \mathrm{q}_{2}^{N}=\left(1-\lambda k_{1} r_{1}-\lambda k_{2} r_{2}\right) \frac{r_{1}^{\prime} r_{2}^{\prime \prime}-r_{2}^{\prime} r_{1}^{\prime \prime}}{\gamma} \\
& \mathrm{q}_{3}^{N}=\dot{\lambda}\left(r_{1} r_{1}^{\prime}+r_{2} r_{2}^{\prime}\right) .
\end{aligned}
$$

Notice that this form is very similar to the form for the Frenet frame, Equations (66)-(68), with the key difference that the centerline curve parameters $k_{1}$ and $k_{2}$ only appear in the binormal velocity $\mathrm{q}_{2}$. This distinction is more apparent 
if the shape $\mathcal{S}$ is a circle so that $r_{1}=\cos (S), r_{2}=\sin (S)$, in which case Equations (66)-(68) become

$$
\begin{aligned}
& \mathbf{q}_{1}^{F}=-\dot{\lambda} \\
& \mathbf{q}_{2}^{F}=1-\lambda \kappa \cos S \\
& \mathbf{q}_{3}^{F}=\lambda \tau
\end{aligned}
$$

and Equations (74)-(76) become

$$
\begin{aligned}
\mathrm{q}_{1}^{N} & =-\dot{\lambda} \\
\mathrm{q}_{2}^{N} & =1-\lambda\left(k_{1} \cos S+k_{2} \sin S\right) \\
\mathrm{q}_{3}^{N} & =0 .
\end{aligned}
$$

In this form, we can understand, in a global sense, the difference between cases II and III for obtaining non-planar growth in Section 3.6. It is apparent that rotating the growth axis (case II) is equivalent to dressing a curve equipped with a natural frame, whereas adding a tangential velocity component is equivalent to dressing a curve equipped with the Frenet frame. Indeed, in the natural frame case, the directors attached to the centerline curve do not twist; hence there is no tangential velocity and the global structure is achieved by varying the binormal velocity in time and space. In the Frenet case, on the other hand, only the curvature of the centerline curve can be captured by binormal growth; torsion (and hence out of plane growth) must be accounted for by tangential growth, which leads to a twist in the centerline curve frame.

\section{Discussion}

In this paper, we have developed a framework for the kinematics of surface growth for objects that can be generated through the evolution of a curve in space. Our analysis relies on the definition of a local orthonormal basis attached to a generating curve, and a growth velocity field defined at every point on the curve and described in terms of the local basis. This formulation provides an intrinsic description of growth, based on local variables. A benefit of the local approach is that the kinematics is directly related to geometry.

A system of differential equations was derived governing the evolution of the generating curve and hence growth of the surface. Several examples were considered to demonstrate the computational steps for solving the system. Obtaining an analytical solution in general seems impractical, however when restricting to the class of surfaces for which the generating curve is planar and maintains a fixed shape, we demonstrated that the system is much more tractable, and in fact fully explicit solutions could be obtained in several cases of biological interest. The assumption that the generating curve is planar and does not change shape is a very common characteristic in shells (Thompson 1942), and is used in nearly all seashell models.

Nevertheless, this is not a requirement of the formulation, and it is certainly desirable to be able to relax the assumption. However, for arbitrary 
cross sections or if the shape of the cross section evolves, the system becomes untractable. Nevertheless, our general geometric framework can be used in a numerical discretisation schem. A proper discretisation of our evolution equations relies on the definition of geometrical quantities such as curvature in a polygonal setting; this connects to the field of research of discrete differential geometry, a field which is largely still in the nascent stage (Bobenko and Suris 2008).

Surface growth is a complex topic, and a full description, even in the case of hard body accretion, requires input from mathematics, mechanics, and biology. The description we have provided here is purely kinematic. Still, from our analysis we can infer several characteristics of the underlying biology. To elaborate, we consider in more detail the growth of a molluscan seashell.

\section{Growth of mollusc seashells}

Growth of mollusc shells is governed by a part of the seashell anatomy called the mantle. The mantle, which is made of soft tissue, attaches to the shell aperture (our generating curve) and controls the deposition of new material. Our analysis enables us to deduce how the deposition of new material might occur. We found in Section 3.5 that the requirement for time invariant self-similar growth is an exponentially increasing growth rate, an unlikely scenario when considering material deposition. Spatially self-similar shell forms, however, can be accomplished by the mantle either maintaining 1) a fixed deposition rate at each point (Fig. 5a), or 2) a deposition rate that is relatively fixed but scales inversely with aperture size (Fig. 5c). The latter may be the most reasonable, as we showed that this form coincides with a fixed material deposition rate, thus corresponding to a constant surface growth rate.

Regarding coiling in shells, we found three different forms of local velocity to produce three-dimensional coiling (what we termed out-of-plane growth). In particular, two of the mechanisms, a rotating growth axis and a tangential velocity, produce identical surfaces, yet are distinctly different from a biological perspective. As stated in Section 3.6, a rotating growth axis corresponds to each point on the aperture keeping the same relative orientation while the location of maximal binormal growth varies with time. With a tangential velocity, on the other hand, each point on the aperture maintains the same relative binormal growth rate, but the aperture itself locally rotates due to a tangential velocity component. Considering the role of the mantle in shell growth, the latter would seem to correspond to each material point of the mantle keeping a fixed relative rate of deposition combined with a rotational component to the mantle. This seems the most reasonable from a biological perspective, and indeed seems to be confirmed in shells where the cell tracks can be traced. For instance, in the Turitella seashell, one can trace the cell tracks by following the locations of the small antimarginal ribs (see Figure 11), and we see that the tracks take the form of Figure 6b, suggesting that the out-of-plane growth was produced by a tangential velocity component.

Closely connected to this issue is the growth axis responsible for coiling. We have shown that linear growth along a fixed axis in the plane of the generating 


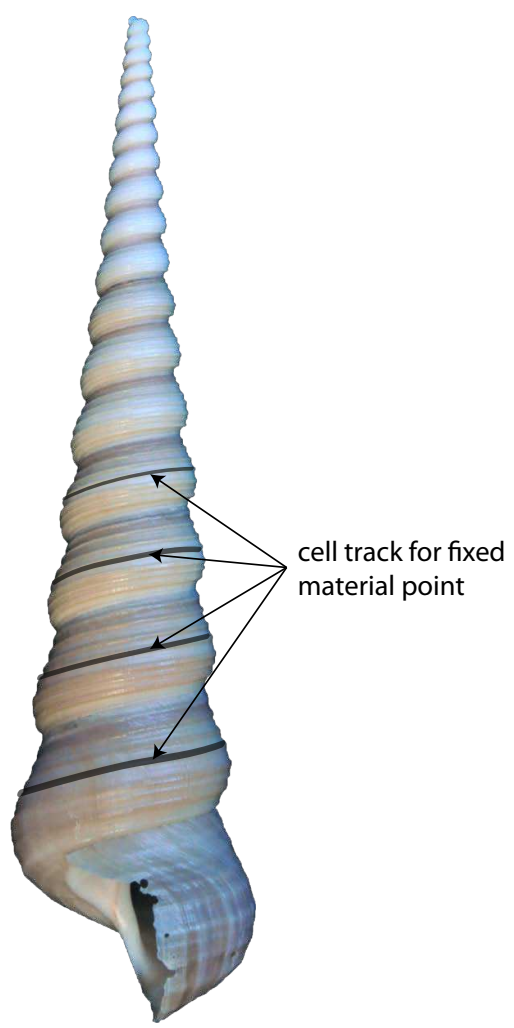

Fig. 11 The "cell tracks" of a Turitella shell can be seen by tracing the small antimarginal ribs. A particular track has been highlighted. The tracks stay in the same relative position on the shell aperture during growth.

curve is necessary if the generating curve is to remain planar, as is the case in the large majority of shells. We can only speculate as to how the growth axis is established and maintained, particularly in cases where the aperture shape is not fixed. In cases where the aperture shape is fixed, it is most likely that the growth gradient is established early in the development, and then maintained throughout the life of the creature. This is most compatible with the idea that each point on the mantle maintains a fixed relative deposition rate, lending further credence to the hypothesis that a tangential velocity is more likely than a rotating growth axis. In cases where the aperture shape evolves, the change in shape is typically temporary, e.g. during the formation of ornamentation such as spines, so the idea of fixed deposition rates is still possible, with a change in mechanism during ornamentation events.

While the mathematical analysis of surface construction can provide useful insight into the mechanisms behind the growth process, the next step is to connect the kinematics description directly to mechanics and/or biology. The notion of mechanotransduction is of particular interest in seashell growth. Since the mantle is elastic tissue and attaches to a rigid body, it is subject to 
stresses and strains, dictated by the local geometry of the aperture. Understanding the interplay between the geometry and the mechanical response is a key step in understanding how growth proceeds. The coupling of kinematics to mechanics in shell growth, as well as the translation of the mathematical description we have given here to a discrete growth environment, forms the subject of a companion paper ${ }^{3}$. Going a step beyond and connecting the mechanics to the biological processes responsible for growth is a challenging task, but an important area of future research.

Acknowledgments: This publication is based on work supported by Award No. KUK-C1-013-04, made by King Abdullah University of Science and Technology (KAUST), and based in part upon work supported by the National Science Foundation under grant DMS-0907773 (AG). AG is a Wolfson/Royal Society Merit Award Holder.

\section{References}

Ackerly S (1989) Kinematics of accretionary shell growth, with examples from brachiopods and molluscs. Paleobiology 15(2):147-164

Antman SS (1995) Nonlinear problems of elasticity. Springer Verlag, New York

Ateshian G (2007) On the theory of reactive mixtures for modeling biological growth. Biomechanics and Modeling in Mechanobiology 6(6):423-445

Bishop RL (1975) There is more than one way to frame a curve. Amer Math Month 82

Black R, Turner S, Johnson M (1994) The early life history of bembicium vittatum philippi, 1846(gastropoda: Littorinidae). Veliger 37(4):393-399

Bobenko A, Suris Y (2008) Discrete differential geometry: integrable structure. Amer Mathematical Society

Boettiger A, Ermentrout B, Oster G (2009) The neural origins of shell structure and pattern in aquatic mollusks. Proceedings of the National Academy of Sciences 106(16):6837

Dera G, Eble G, Neige P, David B (2008) The flourishing diversity of models in theoretical morphology: from current practices to future macroevolutionary and bioenvironmental challenges. Paleobiology 34(3):301

Epstein M (2010) Kinetics of boundary growth. Mechanics Research Communications

Fournier M, Bailleres H, Chanson B (1994) Tree biomechanics: growth, cumulative prestresses, and reorientations. Biomimetics 2

Fowler D, Meinhardt H (1992) Modeling seashells. In: Proc. SIGGRAPH, pp 379-387

Garikipati K (2009) The kinematics of biological growth. Applied Mechanics Reviews 62:030,801

Hammer $\varnothing$, Bucher H (2005) Models for the morphogenesis of the molluscan shell. Lethaia 38(2):111-122

van der Helm A, Ebell P, Bronsvoort W (1998) Modelling mollusc shells with generalized cylinders. Computers \& Graphics 22(4):505-513

Hodge N, Papadopoulos P (2010) A continuum theory of surface growth. Proceedings of the Royal Society A: Mathematical, Physical and Engineering Science 466(2123):3135

Hodge N, Papadopoulos P (2011) Continuum modeling and numerical simulation of cell motility. Journal of Mathematical Biology pp 1-27

3 D.E. Moulton and A. Goriely, Mechanical growth and patterning in seashells, preprint 
Iijima A (2001) Growth of the intertidal snail, monodonta labio (gastropoda, prosobranchia) on the pacific coast of central japan. Bulletin of marine science 68(1):27-36

Meinhardt H (2009) The algorithmic beauty of sea shells. Springer Verlag

Moseley H (1838) On the geometrical forms of turbinated and discoid shells. Philosophical Transactions of the Royal Society of London 128:351-370

Okamoto T (1988a) Analysis of heteromorph ammonoids by differential geometry. Palaeontology 31(pt 1):35-52

Okamoto T (1988b) Developmental regulation and morphological saltation in the heteromorph ammonite Nipponites. Paleobiology 14(3):272-286

Pollack J, Hubickyj O, Bodenheimer P, Lissauer J, Podolak M, Greenzweig Y (1996) Formation of the giant planets by concurrent accretion of solids and gas. ICARUS-NEW YORK- 124:62-85

Raup D (1961) The geometry of coiling in gastropods. Proceedings of the National Academy of Sciences of the United States of America 47(4):602

Raup D, Michelson A (1965) Theoretical morphology of the coiled shell. Science (New York, NY) 147(3663):1294

Rice S (1998) The bio-geometry of mollusc shells. Paleobiology 24(1):133-149

Savazzi E (1987) Geometric and functional constraints on bivalve shell morphology. Lethaia 20(4):293-306

Savazzi E (1990) Biological aspects of theoretical shell morphology. Lethaia 23(2):195-212

Schöne B, Rodland D, Wehrmann A, Heidel B, Oschmann W, Zhang Z, Fiebig J, Beck L (2007) Combined sclerochronologic and oxygen isotope analysis of gastropod shells (gibbula cineraria, north sea): life-history traits and utility as a high-resolution environmental archive for kelp forests. Marine Biology 150(6):1237-1252

Skalak R, Farrow D, Hoger A (1997) Kinematics of surface growth. Journal of mathematical biology 35(8):869-907

Stone J (1996) The evolution of ideas: a phylogeny of shell models. American Naturalist 148(5):904-929

Thompson D (1942) On growth and form. Cambridge, London

Tsui Y, Clyne T (1997) An analytical model for predicting residual stresses in progressively deposited coatings part 1: Planar geometry. Thin Solid Films 306(1):23-33

Tyszka J, Topa P (2005) A new approach to modeling of foraminiferal shells. Paleobiology 31(3):522

Urdy S, Goudemand N, Bucher H, Chirat R (2010) Allometries and the morphogenesis of the molluscan shell: a quantitative and theoretical model. Journal of Experimental Zoology Part B: Molecular and Developmental Evolution 314(4):280-302 



\section{RECENT REPORTS}

30/11 Numerical Study of Liquid Crystal Elastomers by a Mixed Finite Luo

Calderer

31/11 The indentation of pressurized elastic shells: From polymeric cap- Vella sules to yeast cells

Ajdari

Vaziri

Boudaoud

32/11 Wrinkling of pressurized elastic shells

Vella

Ajdari

Vaziri

Boudaoud

33/11 Data assimilation using bayesian filters and B-spline geological models

Duan

Farmer

Hoteit

$\mathrm{Lu}$

Moroz

34/11 Review of nonlinear Kalman, ensemble and particle filtering with application to the reservoir history matching problem

Luo

Hoteit

Duan

Wang

35/11 Modelling a Tethered Mammalian Sperm Cell undergoing Hyperactivation

Curtis

Kirkman-Brown

Connolly

Gaffney

36/11 A simple mathematical model for investigating the effect of cluster roots on plant nutrient uptake

Zygalakis

Roose

37/11 Frequency jumps in the planar vibrations of an elastic beam

Neukirch

Frelat

Goriely

Maurini

38/11 Ice-lens formation and con nement-induced supercooling in soils and other colloidal materials

Style

Cocks

Peppin

Wettlaufer

39/11 An asymptotic theory for the re-equilibration of a micellar surfactant solution

Griffiths

Bain

Breward

Chapman

Howell

Water

40/11 Higher-order numerical methods for stochastic simulation of chemical reaction systems

Székely

Burrage

Erban

Zygalakis

41/11 On the modelling and simulation of a high pressure shift freezing

Smith

Peppin process

Ángel M. Ramos 
45/11 A fibrocontractive mechanochemical model of dermal wound closure incorporating realistic growth factor

Murphy

Hall

Maini

$\mathrm{McCue}$

McElwain

46/11 A two-compartment mechanochemical model of the roles of trans-

Murphy forming growth factor $\beta$ and tissue tension in dermal wound healing

Hall

Maini

McCue

McElwain

47/11 Effects of demographic noise on the synchronization of a metapopulation in a fluctuating environment

Lai

Newby

Bressloff

48/11 High order weak methods for stochastic differential equations based on modified equations

Abdulle

Cohen

Vilmart

Zygalakis

49/11 The kinetics of ice-lens growth in porous media

Style

Peppin

50/11 Wound healing angiogenesis: the clinical implications of a simple

Flegg mathematical model

Byrne

Flegg

McElwain

51/11 Wound healing angiogenesis: the clinical implications of a simple

$\mathrm{Du}$ mathematical model

Gunzburger

Lehoucq

Zhou

52/11 Image Inpainting based on coherence transport with Adapted dis- $\quad$ März tance functions

Copies of these, and any other OCCAM reports can be obtained from:

Oxford Centre for Collaborative Applied Mathematics

Mathematical Institute

24 - 29 St Giles'

Oxford

OX1 3LB

England

www.maths.ox.ac.uk/occam 